\title{
Examining the Comparison of the Outcome Comparison of Nerve Transfer with Different Donor Nerves in a Rat Model
}

\author{
Mustafa Nadi, Rajiv Midha
}

Keywords: Nerve transfer, phrenic nerve, contralateral seventh cervical nerve root, brachial plexus avulsion.

doi:10.1017/cjn.2016.301

Can J Neurol Sci. 2016; 43: 751-752

Brachial plexus injuries (BPIs) have significant physical, psychological and social ramifications on patients and communities. Advances in microsurgical techniques, with appropriate nerve graft and nerve transfer reconstruction, have led to improved outcomes compared to the natural history in many groups of patients. The situation is however more complex in cases of total BPI (TBPI), a condition often associated with multiple nerve root avulsions, as there are only limited surgical options that can be used to reconstruct the BPI. In this context, extraplexal nerve donors such as the contralateral C7 (cC7) spinal nerve root, phrenic nerve (PN), spinal accessory nerve (SAN) and intercostal nerves (ICN) are options that can be used to reinnervate the distal elements of the avulsed BP.

Generally speaking, nerve transfer encompasses the coaptation of a proximal foreign "expendable" donor nerve or branch or fascicle with a distal recipient (denervated by trauma) in an attempt to restore function. It is ideal to mobilize the donor nerve in an attempt to avoid using an interposition nerve graft. This allows one coaptation site which can minimize staggering and axonal loss, with estimates that there may be up to $30 \%$ loss of axons at each coaptation site. ${ }^{1}$ Furthermore, bringing the donor nerve as close as possible to the target end-organ shortens the regeneration distance and hence the recovery time.

As a donor nerve, cC7 was first suggested by Gu et al. in $1986^{2}$ and ever since has been widely used in the Far East. ${ }^{3}$ One main problem with this transfer is the delay and poor overall results because of the long distance that the growing axons have to travel. There are several variations of crossing $\mathrm{cC} 7$ to the injured side: 1) cervical subcutaneous tunnel; 2) prespinal route superficial to scalene anterior muscle (SA); 3) retropharyngealprespinal route between SA and longuscolli muscle; and 4) retropharyngeal prespinal route deep to longuscolli. These modifications progressively aim to eliminate the need or at least shorten the nerve graft length and to create a smoother path in the retroesophageal area. However, there are potential and real complications for the retropharyngeal approaches related to the tunneling through the prespinal route. These include injury and bleeding from the vertebral artery and recurrent laryngeal nerve palsy. ${ }^{3}$

Other complications are related to the dissection of the $\mathrm{cC} 7$, such as injury to the lower trunk that can result in finger and thumb extensor weakness; decreased elbow, wrist, and finger extension strength; pain on the healthy upper limb; atrophy of the sternocostal part of the pectoralis major; ${ }^{3}$ and mild triceps weakness and temporary sensory deficit. ${ }^{4}$ To minimize the morbidity to triceps and sensation, sometimes only part of cC7 (the lateral fascicles - motor fibers to the pectoralis muscle) is used. ${ }^{5}$ Furthermore, the use of $\mathrm{cC} 7$ requires synchronous movement of the opposite normal side to initiate movement, with little or no development of independence over time. Finally, the motor results of cC7 transfer are modest, with MRC Grade 2-3 wrist and finger flexion. When the transfer is to the median nerve, it may give protective hand sensation as well. For all of these reasons, this technique has not become nearly as popular in Western countries as in the Far East.

The phrenic nerve, a motor nerve, which supplies the diaphragm muscle, originates mainly from $\mathrm{C} 4$ spinal nerve root with both $\mathrm{C} 3$ and C5 myotomal contributions. It has several peculiar features that render it an important option to consider as a donor. Firstly, during BPI it can remain intact in $80 \%$ of cases of even the TBPI. ${ }^{5,6}$ Secondly, it contains many pure motor axons that allow the entire or partial transfer with success. It can be use to neurotize musculocutaneous ( $\mathrm{MCN})$, suprascapular nerve (SSN) and axillary nerves with a $75 \%$ success rate. ${ }^{7}$ Furthermore, extensive distal mobilization from the diaphragm using thoracoscopic approaches may allow a direct coaptation to MCN and median nerve recipients in the axilla, with resulting useful motor recovery in many patients. ${ }^{8}$

On the other hand, the downside of sacrificing phrenic nerve is the potential decline of respiratory function, mainly in patients with compromised cardiopulmonary function and in children in general. ${ }^{5}$ Additionally, when the patients attempt to flex the elbow they need to take a deep breath. This requires enormous effort and neural plasticity until the patients is able to eventually adapt. ${ }^{1}$

From the Department of Clinical Neurosciences and Hotchkiss Brain Institute, University of Calgary, Calgary, Alberta, Canada (MN); Department of Clinical Neurosciences and Hotchkiss Brain Institute, University of Calgary, Calgary, Alberta, Canada (RM). Received June 28, 2016. Date of Acceptance July 3, 2016.

Correspondence to: Rajiv Midha, Department of Clinical Neurosciences and Hotchkiss Brain Institute, University of Calgary, Calgary, Alberta, Canada.

Email: rajmidha@ucalgary.ca 
Other nerves that are commonly used for transfer are spinal accessory nerve (SAN) and intercostal nerves (ICN). SAN is isolated and transected distal to its initial trapezius motor branches. It is easily transferred to SSN without interposed graft, or to both SSN and axillary nerves (it needs graft for the latter) and can provide 1500-3000 motor axons. The results of SAN to SSN transfer to restore shoulder abduction are reasonable, but are much better when the axillary nerve can also be re-innervated by a second donor nerve, ${ }^{4}$ which is unfortunately not usually possible because of limited donors in most cases of TBPI. Similarly, ICNs have been successfully transferred to $\mathrm{MCN}$, yet the results are fairly variable and dependent on technical and patient factors. Usually three ICN are used (from $3^{\text {rd }}$ to $5^{\text {th }}$ ) to coapt them directly to distal MCN. In reviewing results of the literature, ICN to $\mathrm{MCN}$ transfer resulted in an average of $72 \%$ of patients achieving bicep muscle strength greater than or equal to anti-gravity strength. ${ }^{5}$

As one can appreciate from the above brief clinical synopsis, the overall outcome of nerve transfer for TBPI is widely variable; at best one can hope to get anti-gravity function in about $50-75 \%$ of patients with shoulder abduction and elbow flexion. With cC7 and phrenic nerve transfers, the possibility of modest hand function with some wrist and finger flexion and protective hand sensation becomes possible in select patients.

Studying and comparing donor nerve type and source of axons appears to be an important factor for understanding the variability in outcomes for reconstruction of TBPI. Although some studies have compared retrospectively reviewed case series of SAN-MCN versus ICN-MCN transfer, ${ }^{5,9,10}$ no study in humans has compared the outcome of using $\mathrm{PN}$ versus $\mathrm{cC} 7$ transfer to re-innervate the BP. Which nerve could be superior to another has not been scientifically evaluated in clinical series, and thus the introduction and detailed study of an animal model is laudable.

In this regard, Jia et al. ${ }^{11}$ present an experimental study which included 60 rats to examine which nerve to opt for $(\mathrm{PN}$ or cC7) to neurotize the upper extremity nerves in BPI. They divided the rats into 3 groups to repair immediate cut injury of radial nerve antebrachial branch (which innervates forelimb wrist and toe extensors): one group is to test efficacy of the PN, another one for cC7 root and a third group as a positive control by cutting and immediate re-approximation of the radial nerve antebrachial branch. They compared the results across the three groups using rigorous tests of behavior, electrophysiology, and myelinated axonal counts, with the conclusion that the $\mathrm{cC} 7$ root is superior to $\mathrm{PN}$ as a donor to neurotize the focal BPI.

The strength of this study lies in establishing a good rodent model for the evaluation of nerve transfers in the upper extremity. Moreover, they specifically compare PN to cC7 transfer, with an appropriate positive control, and present convincing data that for restoration of radial nerve function the $\mathrm{cC} 7$ is superior, but not as good as direct repair. We would advise the authors in the future to extend the model to a more clinically relevant one akin to patients by avulsing all 5 spinal nerve roots of the brachial plexus and then evaluating the outcomes of $\mathrm{PN}$ as compared to $\mathrm{cC} 7$ or other transfers. There are some other concerns that will be difficult to overcome in a rodent model, including major differences in regeneration distances and hence time to re-innervation in human versus rats. In addition, the ulnar nerve graft used in this study to repair cC7 group is short $(4.5 \mathrm{~cm})$. To use contralateral $\mathrm{C} 7$ in humans, the length of the graft is usually much longer and this may have a much more negative impact on the results. Finally, the need for cortical re-learning and plasticity which humans have been shown to (partially) exhibit following these types of nerve transfers ${ }^{12}$ have not been explored in the current paper and could be fruitful to pursue in the future.

\section{Disclosures}

Mustafa Nadi and Rajiv Midha do not have anything to disclose.

\section{REFERENCES}

1. Malessy MJ, Thomeer RT. Evaluation of intercostal to musculocutaneous nerve transfer in reconstructive brachial plexus surgery. Journal of neurosurgery. 1998;88(2):266-71.

2. Gu YD, Zhang GM, Chen DS, Yan JG, Cheng XM, Chen L. Seventh cervical nerve root transfer from the contralateral healthy side for treatment of brachial plexus root avulsion. Journal of hand surgery. 1992;17(5):518-21.

3. Li W, Wang S, Zhao J, et al. Complications of contralateral C-7 transfer through the modified prespinal route for repairing brachial plexus root avulsion injury: a retrospective study of 425 patients. Journal of neurosurgery. 2015;122(6):1421-8.

4. Gu YD, Chen DS, Zhang GM, et al. Long-term functional results of contralateral C7 transfer. Journal of reconstructive microsurgery. 1998;14(1):57-9.

5. Addas BM, Midha R. Nerve transfers for severe nerve injury. Neurosurgery clinics of North America. 2009;20(1):27-38, vi.

6. Chen ZY, Xu JG, Shen LY, Gu YD. Phrenic nerve conduction study in patients with traumatic brachial plexus palsy. Muscle \& nerve. 2001;24(10):1388-90.

7. Songcharoen P. Brachial plexus injury in Thailand: a report of 520 cases. Microsurgery. 1995;16(1):35-9.

8. Gu YD, Ma MK. Use of the phrenic nerve for brachial plexus reconstruction. Clinical orthopaedics and related research. 1996 (323):119-21.

9. Kim DH, Cho YJ, Tiel RL, Kline DG. Outcomes of surgery in 1019 brachial plexus lesions treated at Louisiana State University Health Sciences Center. Journal of neurosurgery. 2003;98 (5):1005-16.

10. Waikakul S, Wongtragul S, Vanadurongwan V. Restoration of elbow flexion in brachial plexus avulsion injury: comparing spinal accessory nerve transfer with intercostal nerve transfer. The Journal of hand surgery. 1999;24(3):571-7.

11. Jia X, Yu C, Yang J. Outcome Comparison of Nerve Transfer with Different Donor Nerves in a Rat Model. The Canadian Journal of Neurological Sciences. 2016.

12. Malessy MJ, Thomeer RT, van Dijk JG. Changing central nervous system control following intercostal nerve transfer. Journal of neurosurgery. 1998;89(4):568-74. 